\title{
Wavelength-dependent nonlinear optical loop mirror for simultaneous amplitude noise reduction at two wavelengths
}

O. Pottiez

pottiez@cio.mx

\section{B. Ibarra-Escamilla}

E. A. Kuzin
Centro de Investigaciones en Óptica, Loma del Bosque 115, Col. Lomas del Campestre, 37150 León, Gto, Mexico

Instituto Nacional de Astrofísica, Óptica y Electrónica (INAOE), L. E. Erro 1, Tonantzintla, 72000 Puebla, Pue., Mexico

Instituto Nacional de Astrofísica, Óptica y Electrónica (INAOE), L. E. Erro 1, Tonantzintla, 72000 Puebla, Pue., Mexico

We propose and study analytically and numerically a polarisation-imbalanced Nonlinear Optical Loop Mirror (NOLM) scheme exhibiting a wavelength-dependent nonlinear characteristic. The device includes a symmetric coupler, an isotropic (or, in practice, twisted nonpolarisation-maintaining) fibre loop and a section of high birefringence (HiBi) fibre to break the polarisation symmetry. Thanks to the wavelength-dependent phase shift of the HiBi fibre, wavelength-dependent switching is obtained. For proper orientation of the HiBi fibre in the loop plane and using linear input polarisation at $45^{\circ}$ with respect to its birefringence axes, the NOLM switching power varies with wavelength, whereas its zero low-power transmission and $100 \%$ maximal transmission are constant. Through slight adjustments of the HiBi fibre birefringence, which can be realised mechanically or thermally, the ratio between switching powers at two particular wavelengths can be readily adjusted. We show numerically that this scheme can be applied to simultaneous amplitude regeneration of two wavelength channels exhibiting uneven power levels.

[DOI: http://dx.doi.org/10.2971/jeos.2013.13059]

Keywords: Nonlinear optical loop mirror, fibre-optic devices, wavelength-selective devices, optical signal regeneration

\section{INTRODUCTION}

As the capacity requirements of optical transmission links grow steadily, multiplexing techniques are being increasingly used to exploit the enormous available bandwidth of optical fibres. These techniques include Wavelength-Division Multiplexing (WDM) and Optical Time-Division Multiplexing (OTDM), which are sometimes combined into hybrid schemes in order to benefit from the advantages of both approaches [1]-[3]. Due to the speed limit of optoelectronic devices, the bit rate of individual data channels is typically limited to 10 Gbit/s, so that it makes sense to interleave them to obtain 40 Gbit/s or more in a single OTDM channel. This creates the need for ultrafast processing devices capable of handling data streams at such high rates. The Nonlinear Optical Loop Mirror (NOLM) [4], which exploits the ultrafast optical Kerr effect in fibre, is a good candidate for many such tasks, and has long been demonstrated for ultrafast switching and demultiplexing [5, 6], wavelength conversion [7], multiplexing format conversion [8], pedestal suppression [9], amplitude equalisation and regeneration of optical signals [10]-[14], etc.

In WDM (and hybrid) systems, where separate channels are encoded through different wavelengths, there is a strong interest in realising functions such as amplitude equalisation and regeneration of different channels using a single fibre device, for obvious cost-saving reasons. Simultaneous multichannel regeneration is a very challenging task, however, which is complicated by the parasitic nonlinear inter- action between channels in a fibre (cross-phase modulation (XPM) and four-wave mixing). In spite of this, solutions were found to mitigate this problem and several efficient fibrebased multiple-wavelength regenerators have been recently proposed [15]-[18], most of them based on nonlinear spectral broadening and offset filtering. This method understandably requires a relatively large wavelength separation between channels, so the need to increase channel spacing [16] is one of a few reasons that explain the inclusion in these schemes of a section where the different channels (or groups of channels) are demultiplexed, processed separately and finally recombined (this final step being unnecessary at the receiver end). Two more reasons that motivate these parallel designs are the use of single-bandpass filters with different central wavelengths for offset filtering each channel [18], and the need for individual adjustment of the power level or timing of each channel [17]. Therefore, in a sense simultaneous regeneration is not performed through a truly single device, as the number of some components (fibre sections, filters, attenuators, delay lines etc) remains proportional to the number of channels.

On the other hand, there is a growing interest to extend the application of the NOLM (and of the related NALM, or nonlinear amplifying loop mirror [19]) towards simultaneous multiwavelength regeneration [20]. In the frame of WDM systems however, where wavelength discrimination is essential, 
the broadband nature of the NOLM nonlinear response restricts its range of applicability. For example, due to the inherent non-uniform gain of fibre amplifiers along an optical link, WDM channels tend to accumulate significant differences between their power levels, which are hardly completely suppressed even if a gain-flattening technique is implemented [21]. If now these channels are affected by amplitude noise, a single device with a specific switching characteristic at each wavelength would allow simultaneous regeneration of different channels, without requiring strict equalisation of power levels among them, or individual power adjustments like in [17].

A natural approach to turn a conventional NOLM into a wavelength-sensitive switch is to include a filtering element in the loop. In [22], this element takes the form of a partially reflecting fibre Bragg grating. Like in most conventional schemes, switching originates from a power imbalance between counter-propagating beams in the loop, with the difference however that this imbalance, introduced here by the grating transmission, is strongly wavelength-dependent. Wavelength-confined switching (switching at the grating wavelength only) was demonstrated experimentally in that work. One drawback of this particular implementation is that the switched signal has to be decoupled from the light reflected by the grating, although such reflections can be avoided through the use of blazed gratings.

Aside from conventional NOLMs, which rely on power imbalance for switching, an alternative scheme was proposed in which a polarisation asymmetry is implemented [23]. The device is power-symmetric, and only the polarisation states of the counter-propagating beams are made different by inserting a birefringent element, namely a quarter-wave retarder (QWR) asymmetrically in the loop. In this case, switching is related to the dependence of the nonlinear phase shift on ellipticity, which is responsible for nonlinear polarisation rotation (NPR). To prevent the nonlinear polarisation evolution from averaging out for each beam, twist is applied to the fibre, which then becomes optically active and behaves like an ideal isotropic fibre [24]. An interesting characteristic of this architecture is the flexibility of the transmission characteristic, which can be adjusted through the control of input polarisation and birefringence. In particular, the dynamic range can be controlled through the QWR angle, with potential applications to large amplitude noise reduction [25]. Besides, controlling the angle of linear input polarisation allows adjusting the switching power, which is attractive for passive mode locking of fibre lasers [26].

As mentioned before, the polarisation-imbalanced NOLM relies on a birefringent element to provide switching. Because the phase shift of a birefringent element, and thus the change of polarisation it causes, is wavelength-dependent, the polarisation asymmetry also varies with wavelength. Hence, in a way similar to the power-imbalanced scheme, in which a wavelength-dependent loss yields wavelengthdependent switching, a polarisation-imbalanced NOLM that includes a strongly birefringent element performing wavelength-dependent polarisation conversion will produce a wavelength-dependent nonlinear characteristic. The scheme that we propose and study in this work is similar to the one proposed in [23], except that the QWR is replaced by a piece of high birefringence (HiBi) fibre cumulating a large number of beat lengths in order to enhance its wavelength dependence. We confirm that switching is wavelength-dependent with this scheme and that, for proper adjustments of input polarisation and NOLM parameters, it can be applied to simultaneous amplitude regeneration of two wavelength channels presenting different power levels.

\section{DEVICE DESCRIPTION AND OPERATION PRINCIPLE}

The proposed scheme is depicted in Figure 1. It includes a 50/50 coupler and the loop mainly consists of isotropic, nonpolarisation-maintaining (non-PM) fibre. Although in practice non-PM fibre always presents some residual birefringence, an isotropic behaviour can still be obtained if twist is applied to the fibre [24]. The device is power-symmetric, and only the symmetry in polarisation is broken by the insertion of a piece of $\mathrm{HiBi}$ fibre at one end of the loop. The HiBi fibre acts as the wave retarder of the polarisation-imbalanced scheme proposed previously [23], with the important difference that here the phase shift between birefringence axes is much larger than $2 \pi$. This phase shift can be slightly adjusted, e.g. mechanically or thermally. Assuming that the coupler does not alter light polarisation, the clockwise (CW) beam enters the loop with the same polarisation as the input beam, and this polarisation state is maintained through the isotropic fibre. In contrast, the polarisation of the counter-clockwise (CCW) beam is modified in general by the HiBi fibre, and the resulting polarisation state is again conserved through the isotropic fibre. Because the optical Kerr effect is polarisation-dependent, this polarisation asymmetry translates into a nonlinear phase shift between the counter-propagating beams as they interfere at the NOLM output, yielding a nonlinear transmission or switching characteristic.

In previous works, a QWR was chosen as the birefringent element, characterised by a $\pi / 2$ phase shift between its slow and fast axes. It is now clear that, for a different value of this phase shift, the polarisation imbalance changes and thus the NOLM switching characteristic will be modified. Considering now that the length $L_{H}$ of the HiBi fibre in the present scheme consists of a large number of beat lengths $L_{B}$, phase shift strongly depends on wavelength. Indeed, at a wavelength $\lambda_{0}$ the phase shift is given by

$$
\Delta \varphi=\frac{2 \pi}{\lambda_{0}} \Delta n L_{H}=2 \pi \frac{L_{H}}{L_{B}}
$$

where $\Delta n$ is the refractive index difference between slow and fast axes (which is assumed to be wavelength-independent). If now the signal wavelength is modified by a small amount $\Delta \lambda<<\lambda_{0}$, this phase shift is modified by

$$
\delta(\Delta \varphi)=-2 \pi \frac{L_{H}}{L_{B}} \frac{\Delta \lambda}{\lambda_{0}}
$$

which may be a substantial fraction of $2 \pi$ if $L_{H} / L_{B}$ is large. In particular, in the $1550 \mathrm{~nm}$ region, for a piece of $\mathrm{HiBi}$ of around 100 beat lengths, a wavelength shift of a few $\mathrm{nm}$ is sufficient 


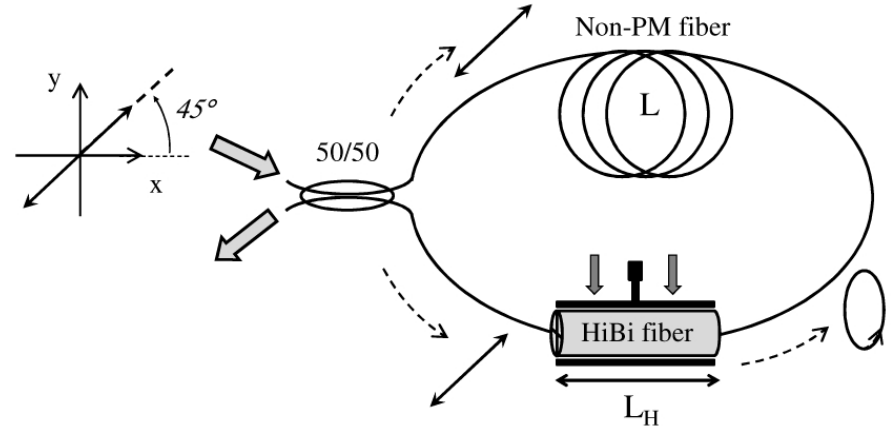

FIG. 1 Configuration under study.

to alter significantly the HiBi fibre phase shift, and thus the NOLM transmission. The nonlinear switching characteristic of the NOLM then becomes wavelength-dependent. In particular, it will be shown in the next section that the NOLM switching power can vary with wavelength.

\section{THEORETICAL ANALYSIS}

In this section we study the nonlinear transmission of the NOLM in the continuous-wave approximation, using the Jones formalism. A comprehensive analysis of the wavelength-dependent NOLM operation considering all possible adjustments of NOLM parameters and input polarisation states would be by far too cumbersome and would divert us from the primary aim of this paper, so we focus on a particular configuration. First, we consider that the orientation of the HiBi fibre axes is fixed at an angle corresponding to zero low-power transmission, as this value ensures maximal dynamic range and optimal regeneration of the " 0 " level. This angle is zero with respect to the plane of the loop if the non-PM fibre is truly isotropic (no twist), and takes a different value for twisted fibre, which amounts to half the total rotatory power [27]. Note that twist-induced optical activity (circular birefringence) in silica fibres is a fraction of the twist whose value $\sim 0.13-0.16$ holds universally [28]. For the sake of simplicity and without loss of generality, it is convenient to consider that twist is adjusted so that the total rotatory power of the non-PM fibre is an exact integer number of turns, ensuring that the HiBi fibre angle for zero low-power transmission is zero. Secondly, we restrict the study to the case of linear input polarisation oriented at $45^{\circ}$ with respect to the $\mathrm{HiBi}$ fibre axes. Linear polarisation is easily obtained in practice through the use of a polariser at the NOLM input, and its orientation at $45^{\circ}$ ensures the smallest value of switching power, as it was shown previously (in the case of a QWR bias) for given fibre length and nonlinearity [27].

In the continuous-wave case (without taking into account dispersion and group velocity mismatch), the nonlinear propagation in the circularly birefringent fibre can be modelled through a simple set of coupled differential equations [23], which in the circular $\left[\mathrm{C}^{+} ; \mathrm{C}^{-}\right]$base write as

$$
\begin{aligned}
& \frac{d C^{+}}{d z}=j \gamma P\left(1-\frac{1}{3} A\right) C^{+} \\
& \frac{d C^{-}}{d z}=j \gamma P\left(1+\frac{1}{3} A\right) C^{-},
\end{aligned}
$$

where $\gamma$ is the nonlinear coefficient of the fibre for linear polarisation, $P=\left|C^{+}\right|^{2}+\left|C^{-}\right|^{2}$ is the optical power and $A=\left[\left|C^{+}\right|^{2}\right.$ $\left.-\left|C^{-}\right|^{2}\right] / P$ is the first Stokes parameter. Although strictly speaking the Stokes parameter in Eqs. (3) varies during propagation, in first approximation it can be viewed as constant for the power ranges considered in this paper (weak nonlinearity limit [23]). In this case, Eqs. (3) are easily integrated over the fibre length and the nonlinear propagation through a length $L$ of fibre can conveniently be modelled as a Jones matrix. If $P_{\text {in }}$ is the NOLM input power, then (considering a lossless 50/50 coupler) the power of both beams that counter-propagate in the loop is $P=P_{i n} / 2$, and if $A_{c w}$ and $A_{c c w}$ are the Stokes parameters of the $\mathrm{CW}$ and $\mathrm{CCW}$ beams, respectively, then the Jones matrix of the fibre in each direction takes the form

$$
\begin{aligned}
& F_{C W / C C W} \\
& \quad=\left[\begin{array}{cc}
e^{j \gamma\left(1-\frac{1}{3} A_{c w / c c w}\right) \frac{P_{i n}}{2} L} & 0 \\
0 & e^{j \gamma\left(1+\frac{1}{3} A_{c w / c c w}\right) \frac{P_{i n}}{2} L}
\end{array}\right] .
\end{aligned}
$$

On the other hand, the Jones matrix of the HiBi fibre whose axes are aligned with the coordinates axes, is given by (in the circular base)

$$
H=\left[\begin{array}{cc}
\cos (\Delta \varphi / 2) & j \sin (\Delta \varphi / 2) \\
j \sin (\Delta \varphi / 2) & \cos (\Delta \varphi / 2)
\end{array}\right]
$$

Now for linear input polarisation at $45^{\circ}$, the input Jones vector in the circular base writes as

$$
E_{i n}=\sqrt{\frac{P_{i n}}{2}}\left[\begin{array}{c}
e^{j \pi / 4} \\
e^{-j \pi / 4}
\end{array}\right],
$$

and the Jones vectors of the counter-propagating beams entering the twisted fibre are given by

$$
\begin{aligned}
E_{c w} & =\frac{1}{\sqrt{2}} E_{i n}=\frac{\sqrt{P_{i n}}}{2}\left[\begin{array}{c}
e^{j \pi / 4} \\
e^{-j \pi / 4}
\end{array}\right] ; \\
E_{c c w} & =\frac{j}{\sqrt{2}} H E_{i n}=\sqrt{\frac{P_{i n}}{2}}\left[\begin{array}{c}
j \cos (\Delta \varphi / 2-\pi / 4) \\
-\sin (\Delta \varphi / 2-\pi / 4)
\end{array}\right] e^{j \pi / 4},
\end{aligned}
$$

where the 50/50 coupler was taken into account by a multiplication by $1 / \sqrt{ } 2$ for the CW field and by $\mathrm{j} / \sqrt{ } 2$ for the CCW field. The Stokes parameter of the $\mathrm{CW}$ and $\mathrm{CCW}$ beams are thus $A_{c w}=0$ and $A_{c c w}=\cos ^{2}(\Delta \varphi / 2-\pi / 4)-\sin ^{2}(\Delta \varphi / 2-\pi / 4)$ $=\cos (\Delta \varphi-\pi / 2)=\sin (\Delta \varphi)$. Knowledge of the Stokes parameters then allows determining the coefficients in the matrixes of the isotropic fibre given in Eq. (4).

The Jones vector at the NOLM output $\mathbf{E}_{\text {out }}$ is obtained by summing the Jones vectors of the CW and CCW beams, which are 
given by

$$
\begin{aligned}
E_{\text {out }, c w} & =\frac{1}{2} H F_{c w} E_{i n} \\
& =\frac{\sqrt{P_{\text {in }}}}{2}\left[\begin{array}{c}
\cos (\Delta \varphi / 2-\pi / 4) \\
j \sin (\Delta \varphi / 2-\pi / 4)
\end{array}\right] e^{j\left(\phi_{n l}+\frac{\pi}{4}\right)} ; \\
E_{\text {out }, c c w} & =-\frac{1}{2} F_{c c w} H E_{i n} \\
& =-\frac{\sqrt{P_{i n}}}{2}\left[\begin{array}{c}
\cos (\Delta \varphi / 2-\pi / 4) e^{j \rho_{n l}} \\
j \sin (\Delta \varphi / 2-\pi / 4) e^{-j \rho_{n l}}
\end{array}\right] e^{j\left(\phi_{n l}+\frac{\pi}{4}\right)},
\end{aligned}
$$

where $\rho_{n l}=\gamma \sin (\Delta \varphi) P_{i n} L / 6$ is the NPR of the CCW beam, and $\phi_{n l}=\gamma P_{i n} L / 2$ is the polarisation-independent nonlinear phase shift. As it appears from Eq. (8), $\phi_{n l}$ is identical for both beams as they carry the same power, so that it cancels out when the two beams interfere at the NOLM output and does not contribute to switching. Instead switching is due to $\rho_{n l}$, which exists only if the ellipticities of the counter-propagating beams are different. The output field is then given by

$$
\begin{aligned}
E_{\text {out }}= & E_{\text {out }, c w}+E_{\text {out }, c c w} \\
= & \sqrt{P_{\text {in }}}\left[\begin{array}{c}
-j \cos (\Delta \varphi / 2-\pi / 4) e^{j \rho_{n l} / 2} \\
-\sin (\Delta \varphi / 2-\pi / 4) e^{-j \rho_{n l} / 2}
\end{array}\right] \\
& \cdot \sin \left(\rho_{n l} / 2\right) e^{j\left(\phi_{n l}+\frac{\pi}{4}\right)} .
\end{aligned}
$$

Finally, the NOLM transmission $T=P_{\text {out }} / P_{\text {in }}$, where $P_{\text {out }}=\left|\mathbf{E}_{\text {out }}\right|^{2}$, is given by

$$
T\left(P_{i n}\right)=\sin ^{2}\left(\rho_{n l} / 2\right)=\frac{1}{2}-\frac{1}{2} \cos \left(\frac{1}{6} \gamma \sin (\Delta \varphi) P_{\text {in }} L\right) .
$$

Eq. (10) is a sinusoidal function of power, whose minimal value is $T(0)=0$ and its maximum $T\left(P_{\pi}\right)=1$, independently of the value of the switching power $P_{\pi}$, which is the power at which $\rho_{n l}$ reaches $\pi$, and is given by

$$
P_{\pi}=\frac{6 \pi}{\gamma L|\sin (\Delta \varphi)|}=\frac{P_{\pi \min }}{|\sin (\Delta \varphi)|},
$$

where $P_{\pi \min }=6 \pi /(\gamma L)$ is the minimal switching power obtained for $|\Delta \varphi|=\pi / 2$. Eq. (11) shows that the switching power depends on the non-PM fibre parameters $\gamma$ and $L$, which determine the value of $P_{\pi \min }$. On the other hand, the presence of $\Delta \varphi$ in Eq. (11) shows that $P_{\pi}$ strongly depends on the polarization state after the HiBi fibre. If for example $\Delta \varphi=2 \mathrm{~m}$ $\pi+\pi / 2$ ( $\mathrm{m}$ being an integer), the CW beam is linearly polarised whereas the CCW beam is circularly polarised in the non-PM fibre, the two beams thus accumulate a strong nonlinear phase shift difference and the switching power is minimal in that case. If now $\Delta \varphi=2 \mathrm{~m} \pi$, both beams are linearly polarised, their nonlinear phase shifts are equal and cancel out, so that no switching is obtained $\left(P_{\pi}=\infty\right)$. As the CW beam in the non-PM fibre is linearly polarised in all cases, switching can be interpreted as arising from the nonlinear phase shift of the CCW beam in the non-PM fibre, which depends on its polarisation state after the HiBi fibre.

If now in Eqs. (10) and (11) we replace $\Delta \varphi$ by $\Delta \varphi+\delta$, and using Eqs. (1) and (2), it appears that the switching power varies with wavelength from a minimal value $P_{\pi \min }$ to infinity, whereas other switching parameters are not altered (in particular the dynamic range, or ratio between maximal

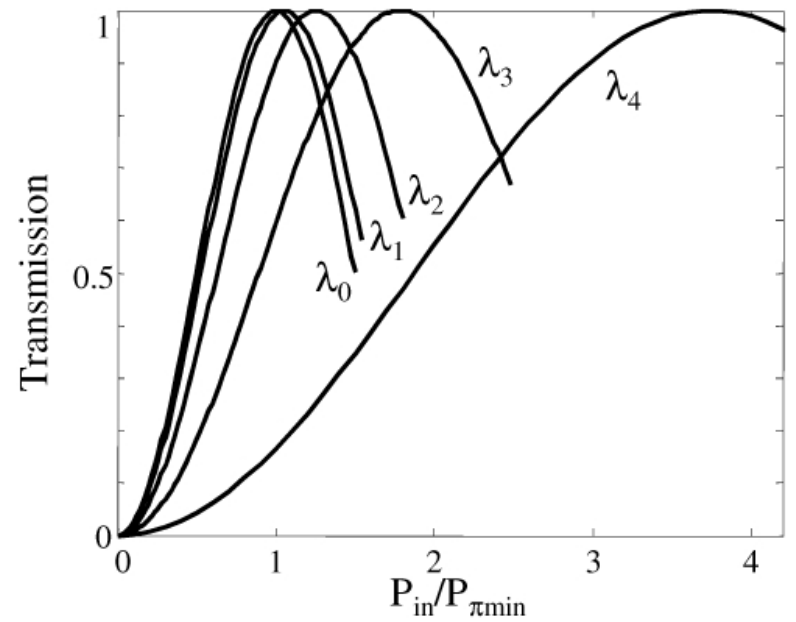

FIG. 2 Wavelength-dependent NOLM nonlinear transmission. In this example, $L_{H} / L_{B}=100.25$ at $\lambda_{0}=1550 \mathrm{~nm}$ and $\lambda_{i}=\lambda_{0}+m \Delta \lambda$ for $m=1$ to 4 , with $\Delta \lambda=0.8 \mathrm{~nm}$ (corresponding to $\delta \approx-\pi / 10$ ).

and minimal transmission, is infinite at any wavelength). This behaviour is illustrated in Figure 2. It appears in this figure that, for a given, constant value of $\Delta \lambda$ (and thus of $\delta$ ) between consecutive curves, a larger switching power ratio $P_{\pi}\left(\lambda_{i+1}\right) / P_{\pi}\left(\lambda_{i}\right)$ between consecutive curves requires larger values of switching power $P_{\pi}\left(\lambda_{i}\right)$.

\section{NUMERICAL STUDY}

In this section we study numerically the operation of the proposed scheme in the frame of amplitude regeneration of a WDM signal consisting of two data trains at different wavelengths and presenting different power levels. When both chromatic dispersion and Kerr nonlinearity are at play, one option consists in operating the NOLM in the soliton regime, which was shown to enhance its regeneration capabilities [29]. However, as the device will process two signals with different power levels and variable power ratio, the pulses at both wavelengths propagating in the loop can hardly be close to fundamental solitons simultaneously, so that the soliton regime cannot be exploited in this case. Therefore we choose for the NOLM loop a highly nonlinear fibre (HNLF) with very low dispersion at $1550 \mathrm{~nm}$. The choice of a HNLF also makes sense as it contributes to reduce the NOLM switching power, which is crucial in the case of densely multiplexed signals, considering their large duty cycle (i.e., the average power at which the device has to be operated is a large fraction of the peak power).

The fibre parameters are $\gamma=10.5 / \mathrm{W} / \mathrm{km}$, second-order dispersion $D=0.3 \mathrm{ps} / \mathrm{nm} / \mathrm{km}$ and dispersion slope $S=0.02 \mathrm{ps} / \mathrm{nm}^{2} / \mathrm{km}$ at $1550 \mathrm{~nm}$. These parameters are typical of commercially available HNLFs. Considering a length $L=200 \mathrm{~m}$ of this fibre in the loop yields $P_{\pi m i n} \approx 9 \mathrm{~W}$ (in the continuous-wave case). In order to compensate for the residual fibre birefringence and obtain an isotropic behaviour [24], a moderate twist of 2 turns / $\mathrm{m}$ is considered. Assuming that the fibre is wound on a spool with a radius of curvature of at least $30 \mathrm{~cm}$, calculations suggest that this twist is sufficient to nearly suppress the effects of linear birefringence, whereas 
it remains small enough to avoid pulse distortion [29]. It has to be noted that the isotropc behaviour of a twisted fibre has been confirmed experimentally. In particular, we found that, using a $\sim 200 \mathrm{~m}$ long piece of standard fiber twisted at 5 turns $/ \mathrm{m}$ and wrapped on a cylinder with a radius of $25 \mathrm{~cm}$, at low power any input ellipticity is maintained at the fibre output. Only when the radius becomes as small as $12 \mathrm{~cm}$ does the linear birefringence of the fibre alter susbtantially the ellipticity. These experimental results thus suggest that, even for moderate twist rates, efficient suppression of the effects of linear birefringence can be obtained if the radius of curvature of the fibre is large enough. Finally, a piece of HiBi fibre whose length corresponds to 32 beat lengths at $1550 \mathrm{~nm}$ (when no pressure/heating is applied) is used (this corresponds to $\sim 10 \mathrm{~cm}$ for a beat length in the few $\mathrm{mm}$ range). Its axes are oriented for zero low-power transmission.

The propagation in the HNLF is modelled using the coupled nonlinear Schrödinger equations [30]. In the base of circular polarisation, these write as

$$
\begin{aligned}
\frac{\partial C^{+}}{\partial z}= & -\frac{\Delta \beta_{1}}{2} \frac{\partial C^{+}}{\partial t}-j \frac{\beta_{2}}{2} \frac{\partial^{2} C^{+}}{\partial t^{2}}+\frac{\beta_{3}}{6} \frac{\partial^{3} C^{+}}{\partial t^{3}} \\
& +\frac{2 j \gamma}{3}\left(\left|C^{+}\right|^{2}+2\left|C^{-}\right|^{2}\right) C^{+}-j \gamma T_{R} \\
& \times\left[\frac{1+\alpha}{2} \frac{\partial}{\partial t} \cdot\left(\left|C^{+}\right|^{2}+\left|C^{-}\right|^{2}\right) C^{+}+(1-\alpha)\right. \\
& \left.\times \frac{\partial}{\partial t}\left(\operatorname{Re}\left(C^{+} C^{-*}\right)\right) C^{-}\right] ; \\
\frac{\partial C^{-}}{\partial z}= & +\frac{\Delta \beta_{1}}{2} \frac{\partial C^{-}}{\partial t}-j \frac{\beta_{2}}{2} \frac{\partial^{2} C^{-}}{\partial t^{2}}+\frac{\beta_{3}}{6} \frac{\partial^{3} C^{-}}{\partial t^{3}} \\
& +\frac{2 j \gamma}{3}\left(\left|C^{-}\right|^{2}+2\left|C^{+}\right|^{2}\right) C^{-}-j \gamma T_{R} \\
& \times\left[\frac{1+\alpha}{2} \frac{\partial}{\partial t}\left(\left|C^{+}\right|^{2}+\left|C^{-}\right|^{2}\right) C^{-}+(1-\alpha)\right. \\
& \left.\times \frac{\partial}{\partial t}\left(\operatorname{Re}\left(C^{+} C^{-*}\right)\right) C^{+}\right] .
\end{aligned}
$$

The first right-hand terms in Eq. (12) account for the group velocity mismatch between circular polarisation components $\left(\Delta \beta_{1}=\beta_{1}^{+}-\beta_{1}^{-}=1 / v_{g}^{+}-1 / v_{g}^{-}\right.$is the inverse group velocity mismatch), the second and third terms describe dispersion $\left(\beta_{2}\right.$ and $\beta_{3}$ are the second- and third-order dispersion parameters, respectively), the fourth terms are Kerr nonlinear terms of selfphase modulation and XPM, where $\gamma$ is the Kerr nonlinear coefficient, and finally the last terms take into account Raman Self-Frequency Shift [31]. $T_{R}$ the Raman delay and $\alpha=0.3$ is the cross-polarisation Raman coefficient at low frequency shift [32]. Time $t$ is referenced in a system that travels at the average group velocity $v_{g}=2 /\left(\beta_{1}^{+}+\beta_{1}^{-}\right)$.

It is important to note that the XPM taken into account in Eq. (12) only refers to the nonlinear interaction between orthogonal polarisation components of the same beam (either $\mathrm{CW}$ or $\mathrm{CCW}$ ), and that the XPM between counter-propagating beams is not taken into account. This effect is substantial in the case of high-duty-cycle multiplexed data streams, and in most cases seriously modifies the NOLM operation [33]. In the present case however, due to circular fibre birefringence, the coefficients of the parallel- and perpendicular-polarisation crossphase terms are equal, so that the XPM experienced by each beam only depends on the opposite beam's power (Chapter 7 in [30]). As powers of CW and CCW beams are equal in this setup, these phase shifts cancel each other. Finally, losses may arise in practice in the scheme of Figure 1, mainly from the splices between non-PM and HiBi fibres. However if low-loss $(<0.1 \mathrm{~dB})$ splices are assumed, fibre loss does not affect very significantly the NOLM operation so that it is neglected in Eq. (12). The Split-Step Fourier technique is used to integrate numerically Eq. (12) for the two beams counter-propagating in the loop. Finally, the wavelength-dependent effect of the HiBi fibre is taken into account by phase-shifting the spectral components of the signal along the $\mathrm{x}$ and $\mathrm{y}$ axes by the wavelength-dependent $\Delta \varphi+\delta$ given by Eqs. (1) and (2).

With the above parameters, Eq. (2) shows that the spectral period of the phase shift of the HiBi fibre (corresponding to a $\delta=2 \pi$ variation) is $\Delta \lambda=48.4 \mathrm{~nm}$. In the following example we consider two signals with central wavelengths at $\lambda_{0}=1550 \mathrm{~nm}$ and $\lambda_{1}=1544 \mathrm{~nm}(|\Delta \lambda|=6 \mathrm{~nm}$, corresponding to $\delta \approx \pi / 4)$. The NOLM nonlinear response at each wavelength was characterised for chirp-free Gaussian input pulses with 11.8 ps full width at half maximum (FWHM) duration and a linear polarisation making a $45^{\circ}$ angle with the $\mathrm{HiBi}$ fibre axes. By adjusting the pressure on the HiBi fibre or through thermal control, the birefringence can be slightly adjusted, and thus the phase shift at $1550 \mathrm{~nm}$ can easily be tuned over the range of a fraction of $2 \pi$ (whereas the value of $\delta$ is nearly unchanged). The curves of Figure 3 show output power at the pulse centre versus input peak power at each wavelength, for different birefringence adjustments. As expected, these curves, and in particular the power at which they reach their maximum, $P_{\max }$, depend on the signal wavelength (note that $P_{\text {max }}$, the power at which $P_{\text {out }}$ saturates, lies slightly beyond the switching power $P_{\pi}$, in the intensity-limiting region of transmission $\left.T=P_{\text {out }} / P_{\text {in }}\right)$.

The most noteworthy feature of Figure 3 is that the ratio between the values of $P_{\max }$ at the two wavelengths varies with the birefringence adjustment: $P_{\max }\left(\lambda_{1}\right) / P_{\max }\left(\lambda_{0}\right)=0.7$ in Figure 3(a), 1.0 in Figure 3(b) and 2.0 in Figure 3(c). This variability can be readily explained by considering Eq. (11), where the phase shift is $\Delta \varphi$ at $\lambda_{0}$ and $\Delta \varphi+\delta=\Delta \varphi+\pi / 4$ at $\lambda_{1}$. Depending on the value of $\Delta \varphi$, the ratio between switching powers $|\sin (\Delta \varphi) / \sin (\Delta \varphi+\pi / 4)|$, and thus the ratio between values of $P_{\max }$, changes. Let us consider in particular that $\Delta \varphi=(2 \mathrm{~m}+3 / 8) \pi, \mathrm{m}$ being an integer, which corresponds to $L_{H} / L_{B}=m+0.1875$ according to Eq. (1). In that case, $\sin (\Delta \varphi)=\sin (\Delta \varphi+\pi / 4)$ and, according to our theoretical model, the switching powers and thus the values of $P_{\max }$ are equal at both wavelengths. The adjustment for which nearly equal curves were obtained numerically in Figure 3(b) $\left(L_{H} / L_{B}=32.17\right)$ is in good agreement with the theoretical value. Finally, it is noticeable that, although the $P_{\max }$ ratio was adjusted over a range from 0.5 to 2 , the smallest of the two values of $P_{\text {max }}$ in each case $(11.1 \mathrm{~W}, 11.7 \mathrm{~W}$ and 9.8 $\mathrm{W}$ respectively) suffers little variations, and does not exceed very significantly the minimal switching power of $9 \mathrm{~W}$ estimated above. Hence, through birefringence adjustments of the NOLM, provided that peak powers are adjusted to the values of $P_{\max }$, it is possible to reduce amplitude noise on the "1" level at two different wavelengths simultaneously for a variable ratio between power levels in each channel, without 

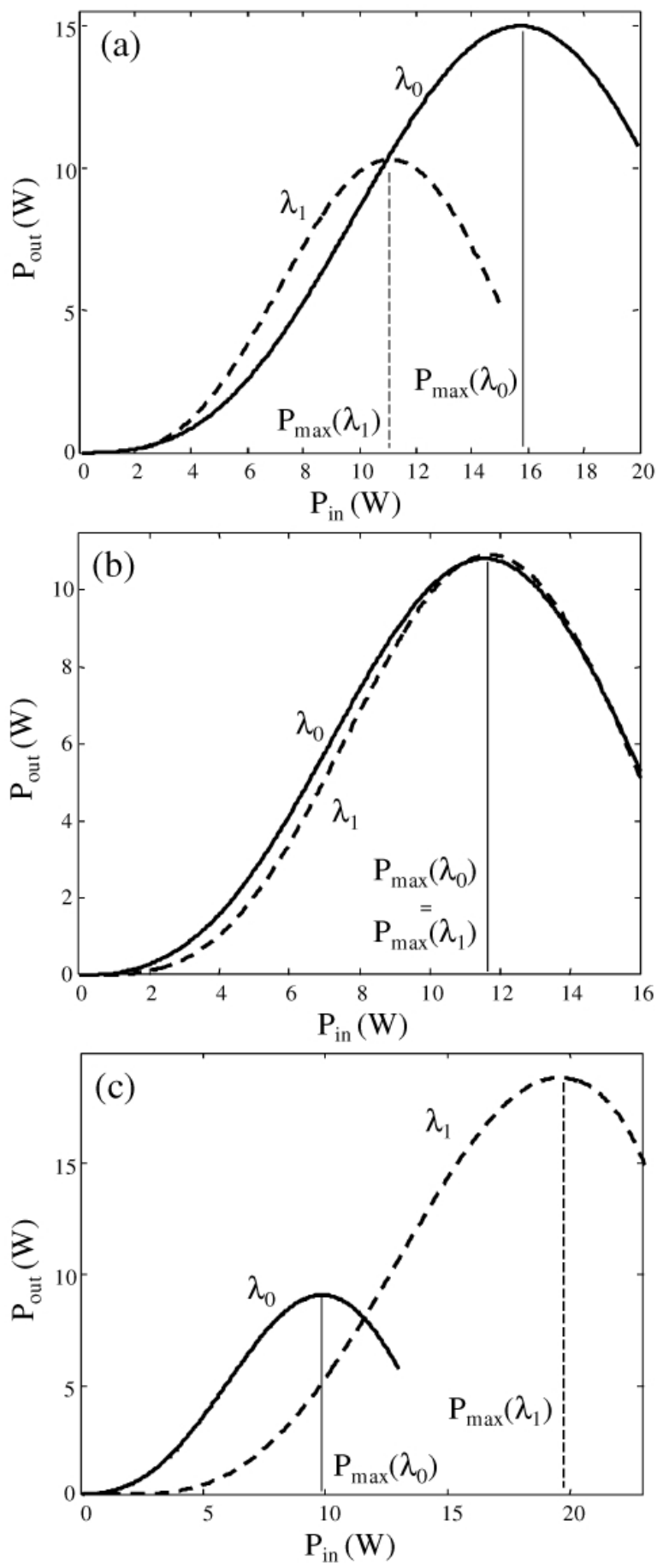

FIG. 3 Output power characteristics of the NOLM at both wavelengths for $L_{H} / L_{B}=32.11$ (a), 32.17 (b) and 32.27 (c).

requiring a dramatic increase in total input power when this power ratio increases moderately. Besides, the very small lowpower transmission of the curves in Figure 3 also allows highquality regeneration of the " 0 " level.

As observed in Figure 3, a drastic change in the ratio between values of $P_{\max }$ at each wavelength can be achieved for a very small variation of the $L_{H} / L_{B}$ ratio (or of the phase shift $\Delta \varphi$ ), which can be realised in pratice through temperature control. On the other hand, such a high sensitivity rises the issue of the temperature stability required to maintain the $P_{\max }$ ratio at a given value. For example, according to our numerical analysis, maintaining $P_{\max }\left(\lambda_{1}\right) / P_{\max }\left(\lambda_{0}\right)$ within $10 \%$ of its desired value requires stabilising $L_{H} / L_{B}$ with a precision of $\sim 0.01$ (or $\Delta \varphi$ with a precision of $\sim 0.01 \times 2 \pi \mathrm{rad}$ ). Taking $L_{H}=32 \mathrm{~mm}$ (for $L_{B}=1 \mathrm{~mm}$ ) and assuming a temperature sensitivity of the HiBi fibre of several $\mathrm{rad} / \mathrm{m} /{ }^{\circ} \mathrm{C}$ [34], this precision requires stabilising the temperature with an accuracy of the order of $0.1^{\circ} \mathrm{C}$. This can readily be achieved in practice by using a Peltier device for temperature control.

The proposed scheme is thus applied to amplitude regeneration of a dual-wavelength (1550 and $1544 \mathrm{~nm}$ ) signal with different power levels at each wavelength. It is assumed that the absolute power level can be adjusted at the NOLM input (using an erbium-doped fibre amplifier, for example), however a relative amplitude difference between the two channels persists. Each signal consists of 11.8-ps FWHM duration pulses with a $10 \%$ Gaussian amplitude noise for the " 1 " level, and ghost pulses with 10 times smaller power and 50\% Gaussian noise for the " 0 " level. Figure 4(a) shows an eye diagram of such a signal in the case when both channels have the same power. An overlap between the pulses at different wavelengths will produce an interference pattern that will prevent proper regeneration, hence if such is the case the signals should be temporally delayed prior to the NOLM input. This can be done using a dispersive element like a chirped grating or a dispersive fibre, for example. We observe proper NOLM operation for a delay of $\sim 2.5$ times the FWHM pulse duration ( $\sim 30$ ps in this case) or higher. For the pulse duration considered here, this means that repetition rates up to 15 $\mathrm{Gb} / \mathrm{s}$ per channel (30 Gb/s in total) can be assumed. Besides, due to the low dispersion of the loop, there is no substantial walkoff between the channels, so that they do not overlap in the loop. As a consequence, nonlinear interactions between the two channels, in particular through XPM and four-wave mixing, which should be carefully managed in many multiwavelength regeneration schemes [15]-[18],[20], do not occur in this case. If now data occupies all possible time slots, as it happens in most telecom appications, then it is not possible to delay the data at both wavelengths and overlap is unavoidable. In this case, careful management of the nonlinear interactions between channels must be implemented, resulting in an increased complexity of the device [20].

The regenerative capabilities of the proposed scheme were tested for different values of the power ratio between channels. Figure 4(b)-(d) illustrates the results for power ratios $P_{\text {peak }}\left(\lambda_{1}\right) / P_{\text {peak }}\left(\lambda_{0}\right)=0.7,1.0$ and 2.0, when the HiBi fibre is adjusted in each case so that the $P_{\max }$ ratio takes these same values, as in Figure 3(a)-(c), respectively. Of course, the input power is also adjusted in each case to ensure that the average peak power of the " 1 " level at each wavelength matches the $P_{\max }$ value of the corresponding switching curve in Figure 3. Proper amplitude regeneration is observed in each case, with significant reduction of the amplitude fluctuations on the " 1 " level at each wavelength, and near cancellation of the power level on the " 0 ".

Besides providing amplitude regeneration of the signal, the NOLM also shortens significantly the pulses. This has long been recognised as a direct consequence of the nonlinear characteristic of NOLMs, which ensures high transmission of the pulse peak but low transmission of its low-power skirts. Aside 

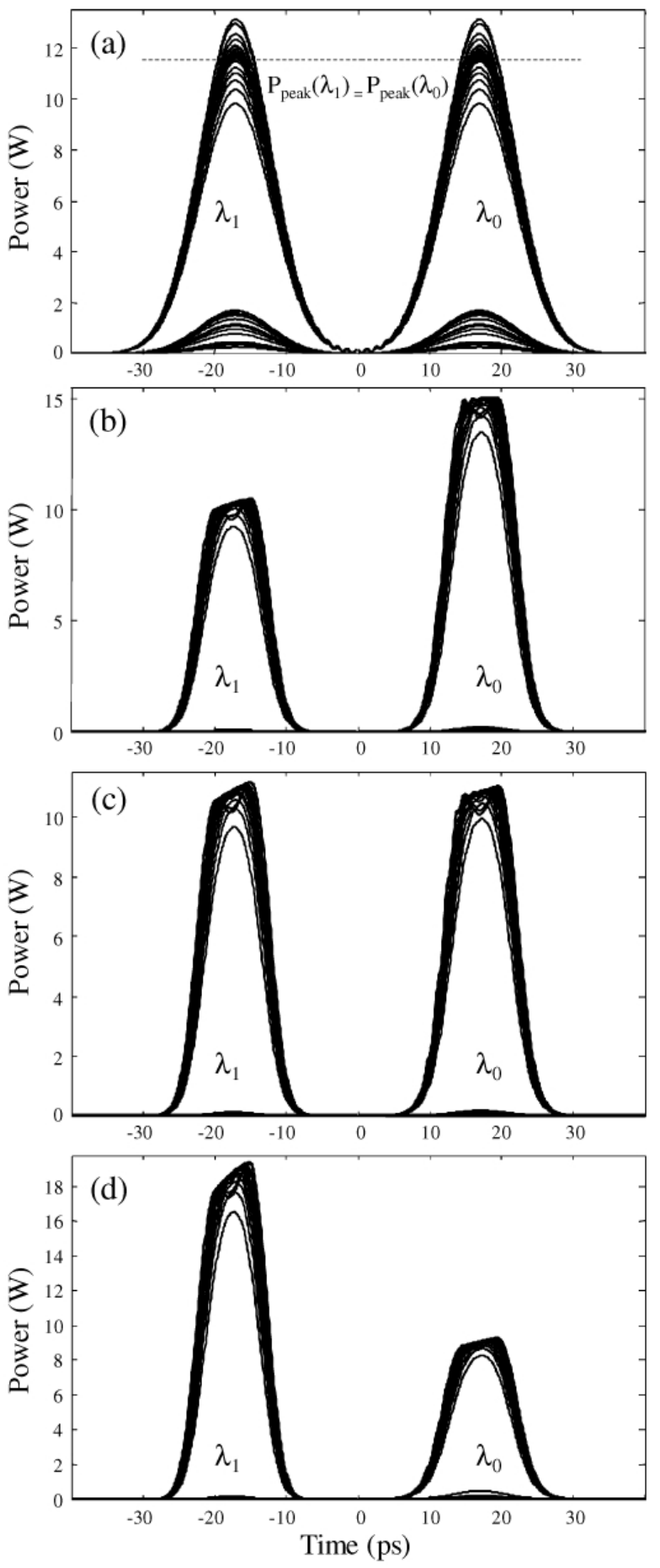

FIG. 4 Optical eye diagrams at the NOLM input for an input peak power ratio $=1.0$ (a), and at the output for input power ratios of 0.7 (b), 1.0 (c) and 2.0 (d). The data repetition rate is $15 \mathrm{~Gb} / \mathrm{s}$ per channel and only amplitude noise is considered (no timing jitter). At the NOLM output, the amplitude noise on the " 1 " level is reduced to $\sim 2 \%$ and ghost pulses are reduced to $\sim 1-2 \%$ of the " 1 " level. Some flattening of the pulses is observed and is related to a slight overdriving of the transmission characteristic beyond the switching point.

from its benefits, the main limitation of the proposed scheme is related to the spectral broadening that originates from the nearly dispersion-free nonlinear evolution in the loop. This broadening sets a lower limit to the wavelength separation between channels. Indeed, if the separation between the two channels considered previously is reduced to, say $1 \mathrm{~nm}$, simultaneous regeneration is still observed in the time domain, however spectral overlap between the two channels at the NOLM output makes it impossible to wavelength demultiplex them. Nonlinear spectral broadening also limits the minimal pulse duration, hence maximal data rate per channel for a given channel separation. The maximal data rate per channel can be improved by a factor of $\sim 2$ or more if temporal overlap between channels in the loop is allowed. This however will give rise to nonlinear interactions whose mitigation would increase the complexity of the scheme [20]. Finally, spectral broadening sets a limit to the power ratio between channels to be regenerated, as a higher power ratio means larger broadening of the high-power signal, even if the level of the lower-power signal remains nearly constant in each case. In future work, we plan to investigate ways to reduce spectral broadening or to decrease the power requirements of the device, through a hybrid power- and polarisation-imbalanced architecture, using a phase bias or through dispersion management, among others.

\section{CONCLUSION}

In summary, we proposed a novel wavelength-sensitive, polarisation-imbalanced NOLM scheme for simultaneous amplitude regeneration of two signals at different wavelengths with different power levels. Polarisation asymmetry and wavelength dependence are obtained through the insertion in the isotropic fibre loop of a piece of HiBi fibre whose length corresponds to a large number of beat lengths. An analytical study of the NOLM transmission showed that, for proper orientation of the $\mathrm{HiBi}$ fibre and linear input polarisation at $45^{\circ}$ with the fibre birefringence axes, the NOLM switching power is wavelength-dependent, whereas other nonlinear transmission parameters like zero low-power transmission and 100\% maximal transmission do not depend on wavelength. We also performed a numerical study, considering for the nonlinear medium a high nonlinearity fibre with slightly anomalous dispersion and a moderated twist to induce isotropy. 32 beat lengths of HiBi fibre were used. The study focused on amplitude regeneration of two signals at different wavelengths with $6 \mathrm{~nm}$ separation and a repetition rate per channel up to $15 \mathrm{~Gb} / \mathrm{s}$. The analysis showed that proper simultaneous regeneration is possible for power ratios from 0.5 to 2, adjusting the average input power and the NOLM birefringence bias mechanically or thermally. Numerical results also suggest that nonlinear spectral broadening sets an upper limit to the power ratio of signals that can be simultaneously regenerated, as well as an upper limit to the ratio between repetition rate and wavelength separation between channels. We believe that this work will pave the way for the design of a range of novel wavelength-sensitive ultrafast processing devices for WDM and hybrid WDM-OTDM systems.

\section{ACKNOWLEDGEMENTS}

O. Pottiez was supported by CONACyT grant 130681 . The authors also thank G. P. Agrawal for fruitful discussions. 


\section{References}

[1] A. A. Aboketaf, L. Cao, D. Adams, A. W. Elshaari, S. F. Preble, M. T. Crowley, L. F. Lester, and P. Ampadu, "Hybrid OTDM and WDM for multicore optical communication," in Proceedings of 2012 International Green Computing Conference (ICCC), 1-5 (IEEE, San Jose, 2012).

[2] Gousia, G. M. Rather, and A. K. Sharma, "WDM-OTDM based spectral efficient hybrid multiplexing technique inherent with properties of bandwidth elasticity and scalability," Optik 121, 1036-1041 (2010).

[3] P. J. Delfyett, B. Mathason, I. Nitta, and H. Shi, “Novel multiwavelength mode-locked semiconductor lasers: physics and applications," Int. J. Hi. Spe. Ele. Syst. 10, 309-317 (2000).

[4] N. J. Doran, and D. Wood, "Nonlinear optical loop mirror," Opt. Lett. 13, 56-58 (1988).

[5] H. Sotobayashi, C. Sawaguchi, Y. Koyamada, and W. Chujo, “Ultrafast walk-off-free nonlinear optical loop mirror by a simplified configuration for 320-Gbit/s time-division multiplexing signal demultiplexing," Opt. Lett. 27, 1555-1557 (2002).

[6] J. H. Lee, T. Tanemura, Y. Takushima, and K. Kikuchi, “All-optical $80-\mathrm{Cb} / \mathrm{s}$ add-drop multiplexer using fiber-based Nonlinear Optical Loop Mirror," IEEE Photon. Technol. Lett. 17, 840-842 (2005).

[7] T. Sakamoto and K. Kikuchi, "160-Gb/s operation of Nonlinear Optical Loop-Mirror with an optical bias controller," IEEE Photon. Technol. Lett. 17, 1058-1060 (2005).

[8] P. Vorreau, F. Parmigiani, M. Ibsen, P. Petropoulos, D. J. Richardson, W. Freude, and J. Leuthold, "TDM-to-WDM conversion based on NOLM from $128.1 \mathrm{Gbit} / \mathrm{s}$ to $3 \times 42.7 \mathrm{Gbit} / \mathrm{s}$," in Proceedings of 2008 ITG Symposium on Photonic Networks, 1-3 (IEEE, Leipzig, 2008).

[9] M. D. Pelusi, Y. Matsui, and A. Suzuki, “Pedestal suppression from compressed femtosecond pulses using a nonlinear fiber loop mirror," IEEE J. Quantum Electron. 35, 867-874 (1999).

[10] M. Attygalle, A. Nirmalathas, and H. F. Liu, "Novel technique for reduction of amplitude modulation of pulse trains generated by subharmonic synchronous mode-locked laser," IEEE Photon. Technol. Lett. 14, 543-545 (2002).

[11] M. Meissner, M. Rösch, B. Schmauss, and G. Leuchs, "12 dB of noise reduction by a NOLM-based 2-R regenerator," IEEE Photon. Technol. Lett. 15, 1297-1299 (2003).

[12] A. Bogoni, P. Chelfi, M. Scaffardi, and L. Potì, "All-optical regeneration and demultiplexing for $160-\mathrm{Cb} / \mathrm{s}$ transmission systems using a NOLM-based three-stage scheme," IEEE J. Sel. Topics Quantum Electron. 10, 192-196 (2004).

[13] S. Boscolo, S. K. Turitsyn, and K. J. Blow, "Nonlinear loop mirrorbased all-optical signal processing in fiber-optic communications," Opt. Fiber Technol. 14, 299-316 (2008).

[14] K. Cvecek, G. Onishchukov, K. Sponsel, A. G. Striegler, B. Schmauss, and G. Leuchs, "Experimental investigation of a modified NOLM for phase-encoded signal regeneration," IEEE Photon. Technol. Lett. 18, 1801-1803 (2006).

[15] T. Ohara, H. Takara, A. Hirano, K. Mori, and S. Kawanishi, "40$\mathrm{Cb} / \mathrm{sx} 4$-channel all-optical multichannel limiter utilising spectrally filtered optical solitons," IEEE Photon. Technol. Lett. 15, 763-765 (2003).

[16] M. Vasilyev, and T. I. Lakoba, "All-optical multichannel $2 R$ regeneration in a fiber-based device," Opt. Lett. 30, 1458-1460 (2005).

[17] Ch. Kouloumentas, P. Vorreau, L. Provost, P. Petropoulos, W. Freude, J. Leuthold, and I. Tomkos, "All-fiberized dispersion- managed multichannel regeneration at $43 \mathrm{~Gb} / \mathrm{s}$," IEEE Photon. Technol. Lett. 20, 1854-1856 (2008).

[18] L. Provost, F. Parmigiani, P. Petropoulos, and D. J. Richardson, "Investigation of simultaneous $2 \mathrm{R}$ regeneration of two $40-\mathrm{Cb} / \mathrm{s}$ channels in a single optical fiber," IEEE Photon. Technol. Lett. 20, 270-272 (2008).

[19] M. E. Fermann, F. Haberl, M. Hofer, and H. Hochreiter, “Nonlinear amplifying loop mirror," Opt. Lett. 15, 752-754 (1990).

[20] T. I. Lakoba, J. R. Williams, and M. Vasilyev, "NALM-based, phasepreserving $2 \mathrm{R}$ regenerator of high-duty-cycle pulses," Opt. Express 19, 23017-23028 (2011).

[21] B. Bakhshi, M. Vaa, E. A. Colovchenko, H. Li, and G. T. Harvey, "Impact of gain-flattening-filter ripple in long-haul WDM systems," in Proceedings of 27th Eur. Conf. On Opt. Comm. (ECOC'01), 448-449 (IEEE, Amsterdam, 2001).

[22] D. A. Pattison, P. N. Kean, W. Forysiak, I. Bennion, and N. J. Doran, "Bandpass switching in a nonlinear-optical loop mirror," 0pt. Lett. 20, 362-364 (1995).

[23] E. A. Kuzin, N. Korneev, J. W. Haus, and B. Ibarra-Escamilla, "Theory of nonlinear loop mirrors with twisted low-birefringence fiber," J. Opt. Soc. Am. B 18, 919-925 (2001).

[24] T. Tanemura and K. Kikuchi, "Circular-birefringence fiber for nonlinear optical signal processing," J. Lightwave Technol. 24, 4108-4119 (2006).

[25] 0. Pottiez, E. A. Kuzin, B. Ibarra-Escamilla, F. Gutiérrez-Zainos, U. Ruiz-Corona, and J. T. Camas-Anzueto, "High-order amplitude regularization of an optical pulse train using a power-symmetric NOLM with adjustable contrast," IEEE Photon. Technol. Lett. 17, 154-156 (2005).

[26] 0. Pottiez, R. Grajales-Coutiño, B. Ibarra Escamilla, E. A. Kuzin, and J. C. Hernandez-Garcia, "Adjustable noise-like pulses from a figure-eight fiber laser," Appl. Opt. 50, E24-E31 (2011).

[27] 0. Pottiez, E. A. Kuzin, B. Ibarra-Escamilla, and F. MendezMartinez, "Theoretical investigation of the NOLM with highly twisted fibre and a $\lambda / 4$ birefringence bias," Opt. Commun. 254, 152-167 (2005).

[28] R. Ulrich, and A. Simon, "Polarization optics of twisted singlemode fibers," Appl. Opt. 18, 2241-2251 (1979).

[29] 0. Pottiez, B. Ibarra Escamilla, and E. A. Kuzin, “Large amplitude noise reduction in ultrashort pulse trains using a power-symmetric Nonlinear Optical Loop Mirror," Opt. ¿t Laser Technol. 41, 384-391 (2009).

[30] G. P. Agrawal, Nonlinear Fiber Optics (Academic Press, San Diego, 2001).

[31] N. Korneev, E. A. Kuzin, B. A. Villagomez-Bernabe, 0. Pottiez, B. Ibarra-Escamilla, A. Conzález-García, and M. Durán-Sánchez, "Raman-induced polarization stabilization of vector solitons in circularly birefringent fibers," Opt. Express 20, 24288-24294 (2012).

[32] I. Mandelbaum, M. Bolshtyansky, T. F. Heinz, and A. R. H. Walker, "Method for measuring the Raman gain tensor in optical fibers," J. Opt. Soc. Am. B 23, 621-627 (2006).

[33] A. Bogoni, M. Scaffardi, P. Chelfi, and L. Potì, "Nonlinear Optical Loop Mirrors: Investigation solution and experimental validation for undesirable counterpropagating effects in all-optical signal processing," IEEE J. Sel. Topics Quantum Electron. 10, 1115-1123 (2004).

[34] F. Zhang, and J. W. Y. Lit, "Temperature and strain sensitivity measurements of high-birefringent polarization-maintaining fibers," Appl. 0pt. 32, 2213-2218 (1993). 